
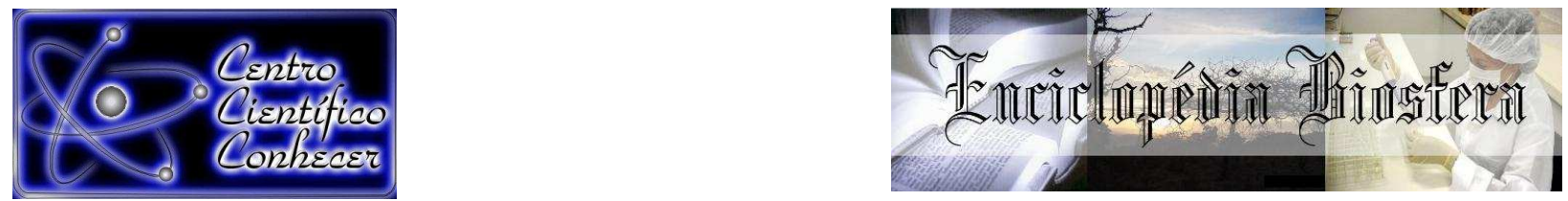

\title{
FLORÍSTICA E FITOSSOCIOLOGIA DE COMUNIDADES DE PALMEIRAS NA RESERVA FLORESTAL HUMAITÁ, ACRE
}

\section{Romário de Mesquita Pinheiro ${ }^{1}$, Evandro José Linhares Ferreira ${ }^{2}$, Ednéia Araújo dos Santos ${ }^{3}$}

1. Graduando em Engenharia Agronômica, Universidade Federal do Acre, Instituto Nacional de Pesquisas da Amazônia - INPA, Rio Branco, Acre

(romario.ufacpz@hotmail.com).

2. Agrônomo, Doutor em Botânica, Pesquisador do Instituto Nacional de

Pesquisas da Amazônia - INPA, Rio Branco, Acre.

3. Engenheira Florestal, Mestre em Botânica, Instituto Nacional de Pesquisas da Amazônia - INPA, Rio Branco, Acre.

Recebido em: 08/09/2015 - Aprovado em: 14/11/2015 - Publicado em: 01/12/2015 DOI: http://dx.doi.org/10.18677/Enciclopedia_Biosfera_2015_086

\begin{abstract}
As palmeiras são importantes componentes das florestas na Amazônia porque apresentam grande variedade de formas de crescimento e são encontradas em todos os estratos florestais, tipos de solos e níveis topográficos. São também abundantes nos ecossistemas onde ocorrem e apresentam grande diversidade de usos e importância sociocultural e econômica, pois possuem frutos comestíveis, estipes, raízes, folhas e outras partes passíveis de algum tipo de aproveitamento. Este estudo avaliou parâmetros fitossociológicos, distribuição, composição, diversidade (Shannon-Wiener) e a similaridade florística (Jaccard) de comunidades de palmeiras em três parcelas medindo $20 \mathrm{~m} \times 100 \mathrm{~m}\left(2.000 \mathrm{~m}^{2}\right)$ instaladas em diferentes gradientes topográficos em área de floresta da Reserva Florestal Humaitá, no Município de Porto Acre, Acre. Foram encontrados 596 indivíduos pertencentes a 12 gêneros e 17 espécies de palmeiras. A diversidade encontrada foi de 1,74, a densidade total 596 ind. ha ${ }^{-1}$ e a área basal 1,684 m2. ha ${ }^{-1}$. Phytelephas macrocarpa apresentou maior densidade e frequência relativa $(38,26 \%$ e $8,33 \%)$ e Euterpe precatoria maior valor de importância $(\mathrm{Vl}=20,37 \%)$. A maioria das espécies $(70,59 \%)$ apresentou padrão de agregação uniforme. As palmeiras da área de platô apresentaram estrutura etária tendendo para 0 ' $J$ ' invertido. A maior similaridade florística foi observada entre as áreas de platô e encosta (70\%), e a menor entre o baixio e a encosta $(63,36 \%)$. A maioria das espécies era desprovida de espinho e apresentou estipe com hábito solitário não escandente. Conclui-se que as palmeiras são mais abundante nos baixios e mais diversa na área de platô.
\end{abstract}

RESUMO

PALAVRAS-CHAVE: Amazônia, Arecaceae, Inventário. 


\title{
FLORISTIC AND PHYTOSOCIOLOGY OF PALM TREES OF COMMUNITIES IN THE HUMAITÁ FOREST RESERVE, ACRE
}

\begin{abstract}
Palm trees are important components of forests in the Amazon region because they present a wide range of growth forms and are found in all forest strata, soils and topographic levels. They are also abundant in the ecosystems where they occur and present a great diversity of uses and socio-cultural and economic importance since they have edible fruits, stems, roots, leaves and other parts subject to some kind of use. This study evaluated phytosociology, distribution, composition, diversity (Shannon-Wiener) and the floristic similarity (Jaccard) of palm communities in three plots measuring $20 \mathrm{~m} \times 100 \mathrm{~m}\left(2,000 \mathrm{~m}^{2}\right)$ installed in different topographic gradients in a forest area of The Humaitá Forest Reserve in the municipality of Porto Acre, Acre. A total of 596 individuals belonging to 12 genera and 17 species of palm trees were found. The diversity index was 1.74 , the density 596 ind. ha $^{-1}$ and the basal area $1,684 \mathrm{~m}^{2}$. ha ${ }^{-1}$. Phytelephas macrocarpa showed higher density and relative frequency $(38.26 \%$ and $8.33 \%$ ) and Euterpe precatoria the highest importance value $(\mathrm{VI}=20.37 \%)$. Most species $(70.59 \%)$ had standard uniform aggregation. The palm community found in the plateau area showed an age structure tending to the reversed ' $J$ '. The greatest floristic similarity was among the palm communities of the plateau and slope areas (70\%), and lowest among the communities in the lowland and hillside areas (63.36\%). Most species presented solitary stem, nonscandent habit, and were spineless. Palm trees are more abundant in the lowland and diverse in plateau areas.
\end{abstract}

KEYWORDS: Amazon, Arecaceae, Inventory.

\section{INTRODUÇÃO}

As palmeiras são uma das famílias botânicas mais importantes da Amazônia em razão de sua ampla distribuição, abundância nos diversos ecossistemas e, principalmente, da diversidade de usos e importância sócio-cultural e econômica de um grande número de espécies nativas (FERREIRA, 2005a; ZAMBRANA et al., 2007; LORENZI et al., 2010). A maioria delas possui algum tipo de utilidade para os habitantes da região (CAMPOS \& EHRINGHAUS, 2003) e isso se deve, principalmente, ao fato da maioria possuir frutos comestíveis, estipes, raízes, folhas e outras partes passíveis de algum tipo de aproveitamento (PINHEIRO, 2011).

De acordo com EISERHARDT et al. (2011) e BALSLEV et al. (2011) as palmeiras são importantes componentes das florestas da Amazônia porque apresentam uma grande variedade de formas de crescimento e podem ser encontrados em todos os estratos florestais, tipos de solos e níveis topográficos. Estão entre as famílias de plantas vasculares dominantes e abundantes nas florestas neotropicais, amplamente distribuídas por toda a Amazônia, algumas vezes formando extensas florestas oligárquicas (PETERS et al., 1989; TER STEEGE et al., 2013; SANTOS et al., 2014; EMILIO et al., 2014). As palmeiras também são eficientes na colonização e sobrevivência em novos habitats, especialmente naqueles alterados pelo homem (MARTINS, 2012).

A Amazônia abriga a maior diversidade de palmeiras do Brasil, com 35 dos 42 gêneros e cerca de 150 das 193-208 espécies reconhecidas para o país 
(HENDERSON, 1995; LORENZI et al., 2010; SOUSA \& LORENZI, 2012). Segundo FERREIRA (2005a), os gêneros mais diversificados são Bactris e Geonoma, que representam cerca de $43 \%$ das espécies amazônicas. Astrocaryum, Attalea, Oenocarpus, Syagrus, Desmoncus, Euterpe e Leopoldinia são outros gêneros importantes que correspondem a cerca de 30\% das espécies nativas. Estes números sugerem que cerca de $25 \%$ dos gêneros nativos são responsáveis por mais de $70 \%$ das espécies encontradas na região.

Apesar dos recentes avanços sobre o conhecimento da diversidade de palmeiras da Amazônia brasileira (FERREIRA, 2005b; HENDERSON, 1995; LORENZI et al., 2010), ainda não se pode afirmar que a flora das palmeiras amazônicas seja bem conhecida, pois existem extensas áreas da região com pouca ou nenhuma amostragem de palmeiras. HENDERSON (1995) afirma que faltam amostras de aproximadamente metade da área da Amazônia.

No contexto atual de antropização e destruição continuada de extensas áreas florestais na Amazônia sabe-se que as comunidades de palmeiras estão sendo afetadas negativamente. Indiretamente isto pode afetar toda a floresta, pois as palmeiras, presentes por toda a região, funcionam como recurso-chave para a fauna e milhares de famílias de agricultores, especialmente no período de escassez de frutos (LORENZI et al., 2010; MESQUITA et al., 2014).

Para determinar os efeitos das transformações ambientais sobre as comunidades de palmeiras da região são necessários estudos para conhecer a diversidade, riqueza, distribuição e estrutura dessas comunidades. Entretanto, a maioria dos estudos abordando a distribuição e estrutura de espécies florestais na Amazônia tem focado nas espécies arbóreas utilizando critérios de amostragem $(D A P \geq 10 \mathrm{~cm}$ ) que excluem grande parte das palmeiras nativas, que em sua maioria apresentam DAP menor que $10 \mathrm{~cm}$ (ROCHA \& SILVA, 2005). Isso é particularmente significativo no caso das espécies de pequeno porte, a maioria pertencente aos gêneros Bactris e Geonoma, os mais diversos na região. Esta deficiência de informações dificulta a implementação de ações voltadas para a exploração e o manejo sustentado dos recursos oferecidos pelas palmeiras, pondo em risco a preservação das espécies, do estoque genético que elas representam e a conservação dos habitats que elas ocupam (MIRANDA \& RABELO, 2008).

Em virtude do exposto, o objetivo desse trabalho foi avaliar a distribuição, composição e a diversidade florística, parâmetros fitossociológicos e a similaridade florística de comunidades de palmeiras em diferentes gradientes topográficos na Reserva Florestal Humaitá, no Município de Porto Acre, Acre.

\section{MATERIAL E MÉTODOS}

\section{Caracterização da área de estudo}

O estudo foi realizado na Reserva Florestal Humaitá, uma unidade de pesquisa pertencente à Universidade Federal do Acre localizada no município de

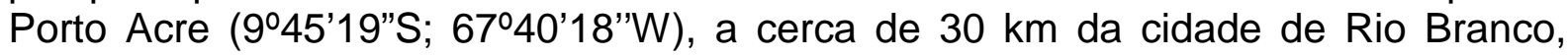
capital do Estado. A reserva possui uma área aproximada de 2.000 hectares quase completamente coberta por Floresta Tropical Aberta com palmeiras ou bambu no subosque, e em menor escala Floresta Tropical Densa e Floresta de Várzea na área que margeia o rio Acre (ACRE, 2006).

O relevo local é suave-ondulado e encontra-se cortado por vários igarapés que inundam áreas florestais nas regiões mais baixas do terreno durante o período 
chuvoso. A precipitação pluviométrica média anual é de $1940 \mathrm{~mm}$, observando-se um período com chuvas mais intensas entre janeiro e março e uma estiagem pronunciada entre julho e agosto. A temperatura média anual é de $26^{\circ} \mathrm{C}$. Entretanto quedas bruscas de temperatura, com mínimas inferiores a $10^{\circ} \mathrm{C}$, ocorrem durante $\mathrm{O}$ período seco em razão do o avanço de frentes polares que causam um fenômeno conhecido localmente como "friagem" (DUARTE, 2005).

\section{Alocação da parcela para o estudo florístico e informações coletadas}

Para a amostragem fitossociológica foi empregado o método de parcelas (MUELLER-DOMBOIS \& ELLENBERG, 1974). Foram instaladas três parcelas em uma área florestal de terra firme (RIBEIRO et al., 1999) sobre relevo ondulado e sem a presença de bambu (Guadua spp.) no subosque. Cada parcela tinha $20 \mathrm{~m}$ de largura e $100 \mathrm{~m}$ de comprimento (totalizando $2.000 \mathrm{~m}^{2}$ ), perfazendo uma área amostral total de $6.000 \mathrm{~m}^{2}$. Para facilitar a coleta dos dados, as parcelas foram subdivididas em cinco sub-parcelas de $20 \times 20 \mathrm{~m}\left(400 \mathrm{~m}^{2}\right)$. Para avaliar o efeito do gradiente topográfico sobre a composição, diversidade e similaridade florística, estrutura populacional e horizontal das palmeiras, as três parcelas foram instaladas, respectivamente, em áreas de floresta de baixio, em floresta de encosta ou vertente e floresta de platô. As florestas de platô situam-se nas áreas mais planas e elevadas do terreno, geralmente sobre solos mais argilosos. As áreas de baixio, localizadas nas áreas mais baixas do terreno, estão associadas a cursos de água (igarapés) e apresentam solos mais arenosos, sendo inundadas sazonalmente no período das chuvas. As florestas situadas em áreas de encosta ou de vertente são consideradas uma transição entre as áreas de platô e baixio e apresentam solos mais argilosos na parte mais alta e areno-argilosos na parte mais baixa (GOULDING et al., 1988; HOPKINS, 2005).

Em cada parcela foram registradas as espécies de palmeiras presentes e avaliados a altura, diâmetro na base do estipe (espécies de pequeno porte) e diâmetro a altura do peito-DAP (espécies de grande porte), número de folhas, presença/ausência de frutos e flores. Palmeiras que formam touceiras (com mais de um estipe) foram consideradas como um único indivíduo. Para as palmeiras acaulescentes, não foram tomadas medidas do estipe (SILVA et al., 2014).

Para avaliar a estrutura populacional, todas as palmeiras encontradas no interior das parcelas foram classificadas em cinco classes de tamanho, conforme o procedimento adotado por CARVALHO et al. (2010): classe 1: indivíduos com até 50 cm de altura; classe 2: indivíduos com altura entre 0,5 e 1,0 m, sem estipe aparente; classe 3: indivíduos com mais de $1 \mathrm{~m}$ de altura e sem estipe aparente; classe 4: indivíduos com estipes aparentes que ainda não atingiram o estágio reprodutivos; classe 5: indivíduos adultos que já atingiram o estágio reprodutivo.

\section{Dados florísticos e fitossociológicos}

Foram avaliadas a composição florística e o índice de diversidade florística de Shannon-Wiener $\left(\mathrm{H}^{\prime}\right)$ de cada uma das áreas, assim como determinado o índice de similaridade florística (Jaccard) entre as mesmas, e o índice de agregação das espécies (IGA) (BROWER \& ZARR, 1984; MAGURRAN, 1988). O parâmetro fitossociológico avaliado foi a estrutura horizontal. A identificação botânica das espécies de palmeiras encontradas na área foi realizada in loco por meio do reconhecimento de suas características dendrológicas por especialista na família 
(Dr. Evandro Ferreira) e no herbário mediante o uso de bibliografia especializada (HENDERSON, 1995; HENDERSON et al., 1995; LORENZI et al., 2010).

\section{Aspectos morfológicos das palmeiras}

Todas as espécies de palmeiras encontradas durante o levantamento foram classificadas quanto ao hábito (solitário ou cespitoso) e tipo de crescimento do estipe (escandente e não escandente), conforme o procedimento adotado por BALSLEV et al. (2010). A presença de espinhos foi anotada tendo em vista a importância dos mesmos na defesa das palmeiras contra herbívoros (BAKER \& COUVREUR, 2013).

\section{Análises dos dados florísticos e fitossociológicos}

A tabulação dos dados foi feita no software Microsoft Office Excel 2007. A análise dos dados de composição florística e dos parâmetros fitossociológicos foi feita com auxílio do software Mata Nativa versão 3.03, desenvolvido na Universidade Federal de Viçosa (SOUZA, 2006).

\section{RESULTADOS E DISCUSSÃO}

\section{Composição Florística Geral}

Foram encontrados 596 indivíduos pertencentes a 12 gêneros e 17 espécies de palmeiras (Quadro 1), sendo que três indivíduos (Attalea sp., Geonoma sp. e Bactris sp.) foram identificadas apenas no nível de gênero. Os gêneros mais diversos foram Bactris, com quatro espécies, seguido de Attalea e Geonoma, com duas espécies cada. Apesar de avaliarem áreas com dimensões diferentes, CARVALHO et al. (2010) identificaram 19 espécies e 12 gêneros de palmeiras em um fragmento florestal primário e secundário na Área de Proteção Ambiental (APA) Raimundo Irineu Serra, nas cercanias de Rio Branco, números aproximados aos observados no presente estudo.

\section{Composição florística na área de baixio}

$\mathrm{Na}$ área de baixio foram encontrados 309 indivíduos, pertencentes a 15 espécies e 12 gêneros, e que correspondem a 51,84\% dos indivíduos amostrados (quadro 1). O único gênero diversificado foi Bactris, com quatro espécies.

As espécies mais abundantes foram Phytelephas macrocarpa (175 indivíduos), Astrocaryum ulei (45 indivíduos) e Euterpe precatoria (39 indivíduos), que juntas correspondem a $83,81 \%$ dos indivíduos amostrados. As espécies menos abundantes foram Aiphanes aculatea, Bactris concinna, Bactris major, Bactris sp., Geonoma acaule, Geonoma sp., com apenas um individuo cada. 
QUADRO 1 - Ocorrência de espécies de palmeiras em áreas de baixio, encosta e platô em floresta sem bambu da Reserva Florestal Humaitá, Porto Acre, Acre.

\begin{tabular}{lccc}
\hline Nome científico & Baixio & Encosta & Platô \\
\hline Aiphanes aculeata Willd. & $\checkmark$ & & \\
Astrocaryum ulei Burret & $\checkmark$ & $\checkmark$ & $\checkmark$ \\
Attalea phalerata Mart. ex Spreng. & $\checkmark$ & $\checkmark$ & $\checkmark$ \\
Attalea sp. & & $\checkmark$ & $\checkmark$ \\
Desmoncus mitis Mart. & $\checkmark$ & $\checkmark$ & $\checkmark$ \\
Euterpe precatoria Mart. & $\checkmark$ & $\checkmark$ & $\checkmark$ \\
Geonoma acaule Mart. & & & $\checkmark$ \\
Geonoma sp. & $\checkmark$ & & \\
Oenocarpus mapora H.Karst. & $\checkmark$ & $\checkmark$ & $\checkmark$ \\
Phytelephas macrocarpa Ruiz \& Pav. & $\checkmark$ & $\checkmark$ & $\checkmark$ \\
Socratea exorrhiza (Mart.) H.Wendl. & $\checkmark$ & $\checkmark$ & \\
Iriartea deltoidea Ruiz \& Pav. & $\checkmark$ & & \\
Bactris concinna Mart. & $\checkmark$ & & $\checkmark$ \\
Bactris maraja Mart. & $\checkmark$ & $\checkmark$ & $\checkmark$ \\
Bactris sp. & $\checkmark$ & & $\checkmark$ \\
Bactris major Jacq. & $\checkmark$ & & \\
Chelyocarpus chuco (Mart.) H.E.Moore & $\checkmark$ & $\checkmark$ & \\
\hline
\end{tabular}

SILVA et al. (2014), avaliando as comunidades de palmeiras em diferentes gradientes topográficos da APA do Igarapé São Francisco, em Rio Branco, Acre, encontraram 308 indivíduos de palmeiras identificados em 11 gêneros e 15 espécies em área de baixio em floresta ombrófila aberta sem bambu (Guadua spp.).

\section{Composição florística na área de encosta}

$\mathrm{Na}$ área de encosta foram encontrados 151 indivíduos, pertencentes a nove espécies e nove gêneros (quadro 1), que correspondem a $24,64 \%$ dos indivíduos amostrados. Nesta área nenhum gênero apresentou mais de uma espécie. As espécies mais abundantes foram Astrocaryum ulei e Attalea phalerata, com 57 e 48 indivíduos, correspondendo a $69,53 \%$ dos indivíduos amostrados. As espécies menos abundantes foram Chelyocarpus chuco, com apenas um individuo, e Oenocarpus mapora e Socratea exorrhiza, com dois indivíduos cada.

Em área de encosta na APA Igarapé São Francisco, em Rio Branco, Acre, SILVA et al. (2014), encontraram 144 indivíduos classificados em nove gêneros e 11 espécies de palmeiras em floresta ombrófila aberta sem bambu (Guadua spp.).

\section{Composição florística na área de platô}

No platô foram encontrados 136 indivíduos, pertencentes a 11 espécies e oito gêneros. As espécies mais abundantes foram Phytelephas macrocarpa (40 indivíduos) e Astrocaryum ulei e Euterpe precatoria (31 indivíduos cada), que juntas correspondem a $75 \%$ dos indivíduos amostrados (Tabela 1). Na APA Igarapé São Francisco, em Rio Branco, Acre, SILVA et al. (2014), encontraram 79 indivíduos classificados em nove gêneros e 11 espécies de palmeiras em área de platô em floresta sem bambu (Guadua spp.) no subosque. 
TABELA 1-Ocorrência e número de indivíduos por espécie de palmeira nas subparcelas instaladas nas áreas de baixio, encosta e platô em área de floresta sem bambu (Guadua spp.) na Reserva Florestal Humaitá, Porto Acre, Acre.

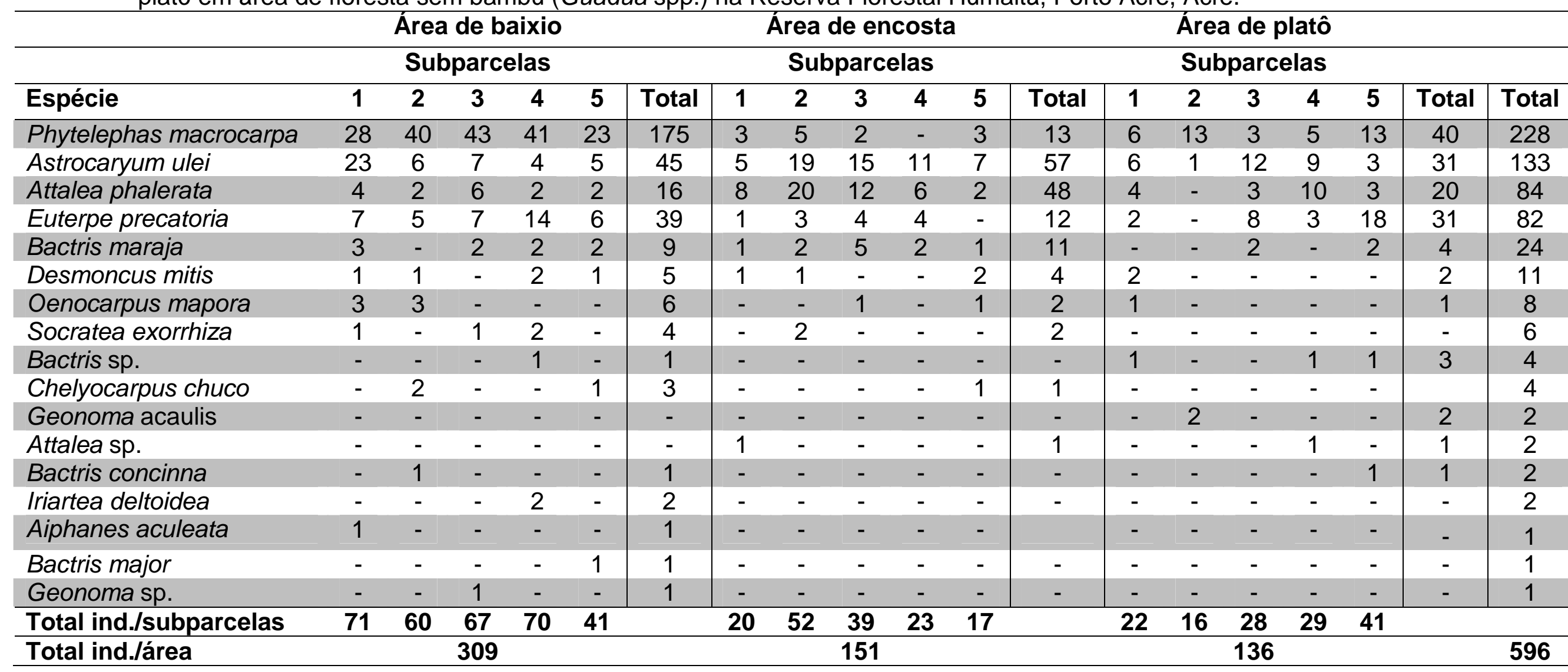


O gênero mais diversificado foi Bactris, com três espécies, das quais uma delas foi identificada apenas ao nível de gênero. Attalea apresentou duas espécies, sendo uma delas identificada apenas ao nível de gênero. As espécies mais abundantes foram Phytelephas macrocarpa (44 indivíduos), Euterpe precatoria (34 indivíduos) e Astrocaryum ulei (33 indivíduos), que juntos correspondem a 65,29\% dos indivíduos amostrados. As espécies menos abundantes foram Oenocarpus mapora e Bactris concinna, cada uma com apenas um indivíduo.

\section{Diversidade Florística}

A diversidade florística para as três áreas foi de 1,74 , sendo que a diversidade foi maior na área de platô $\left(H^{\prime}=1,74\right)$, seguida da área de encosta $\left(H^{\prime}=1,61\right)$ e área de baixio $\left(H^{\prime}=1,49\right)$. Apesar de baixo, esse valor do índice de diversidade encontrado é mais elevado do que o encontrado por CARVALHO et al. (2010) na APA Irineu Serra, nas cercanias de Rio Branco, Acre, que foi de 1,65.

\section{Estrutura populacional}

A classificação dos indivíduos amostrados na área de baixio, encosta e platô quanto à classe de tamanho foi a seguinte: $37,78 \%$ na classe $1,15,77 \%$ na classe 2 , $22,99 \%$ na classe $3,11,91 \%$ na classe 4 e $11,58 \%$ na classe 5 (Figura $1 \mathrm{~A}$ ).

A classificação dos indivíduos amostrados na área de baixio quanto à classe de tamanho foi a seguinte: $19,41 \%$ na classe $1,19,09 \%$ na $2,33,65 \%$ na $3,13,26 \%$ na 4 , e $14,56 \%$ na classe 5 (Figura 1B). Na área de encosta o resultado foi o seguinte: $60,26 \%$ dos indivíduos na classe $1,9,93 \%$ na $2,9,93 \%$ na $3,16,55 \%$ na 4 , e 3,31\% na classe 5 (Figura 1C). Na área de platô foi observado o seguinte: $54,41 \%$ na classe $1,14,70 \%$ na $2,13,23 \%$ na $3,67 \%$ na 4 , e 13,97\% na classe 5 (Figura 1D).
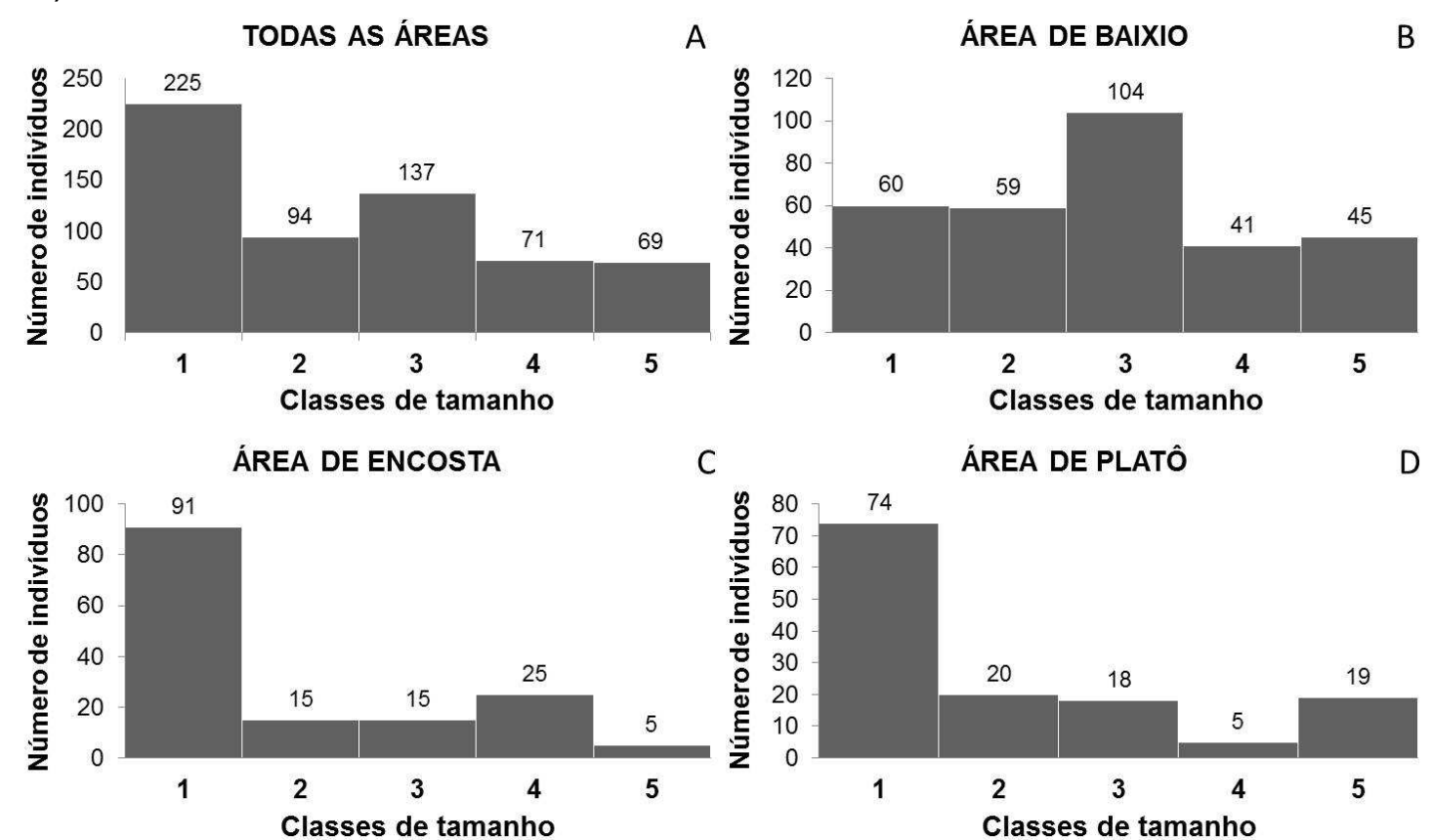

FIGURA 1 - Estrutura populacional das palmeiras na Reserva Florestal Humaitá, Acre: A) Estrutura populacional de todas as áreas; B) Estrutura populacional da área de baixio; C) Estrutura populacional da área de encosta; D) Estrutura populacional da área de platô. Classes de tamanho: 1 (até $0,5 \mathrm{~m}$ de altura); 2 (+0,5 $\mathrm{m}$ até $1,0 \mathrm{~m}$ de altura); 3 ( $+1,0 \mathrm{~m}$ de altura, sem estipe aparente); 4 (com estipe aparente, não atingiram estágio reprodutivo); 5 (adultos, em estágio

reprodutivo). 


\section{Estrutura horizontal}

A densidade total em todas as parcelas foi de 596 ind.ha $^{-1}$ e a área basal $1,684 \mathrm{~m}^{2} \cdot \mathrm{ha}^{-1}$ (Tabela 2). As espécies que apresentaram as maiores densidade $\mathrm{e}$ frequência relativas foram Phytelephas macrocarpa (38,26\% e 8,33\%) e Astrocaryum ulei (22,32\% e 8,33\%). As espécies com maior valor de importância foram Euterpe precatoria ( $\mathrm{VI}=20,37 \% ; \mathrm{N}=82$ ) e Astrocaryum ulei ( $\mathrm{VI}=20,13 \%$ $\mathrm{N}=133$ ). SILVA et al. (2014) também encontraram que $E$. precatoria era a espécie com maior valor de importância em seu estudo sobre comunidades de palmeiras na APA Igarapé São Francisco.

Os valores de densidade e frequência relativa de Phytelephas macrocarpa e Astrocaryum ulei podem ser explicados pela presença de vários indivíduos adultos próximos as parcelas instaladas. No caso de Phytelephas macrocarpa, uma espécie acaulescente do subosque da floresta, existe uma população adensada da espécie (conhecida pelos moradores locais como 'jarinal') na área de baixio, onde os solos são mais úmidos e a tipologia florestal é do tipo 'Floresta Aberta com Palmeira no Subosque'. Além disso, observa-se que as árvores emergentes do local apresentam comportamento caducifólio, perdendo as folhas no período seco, o que favorece a entrada de luz até o solo da floresta, condição que favorece, no sudoeste da Amazônia, a dominância das áreas de subosque florestais pelas palmeiras e pelo bambu (Guadua spp.) (FERREIRA, 2014). 
TABELA 2 - Relação das espécies amostradas nas áreas de baixio, encosta e platô de floresta sem bambu (Guadua spp.) no subosque na Reserva Florestal Humaitá em Porto Acre, Acre, com seus respectivos parâmetros fitossociológicos. $\mathrm{N}=$ número de indivíduos; $\mathrm{U}=$ parcela; $\mathrm{AB}=$ área basal; $\mathrm{DA}=$ densidade absoluta; $\mathrm{DR}=$ densidade relativa; $\mathrm{FA}=$ frequência absoluta; FR=frequência relativa; DoA=dominância absoluta; DoR=dominância relativa; VC=valor de cobertura;

$\mathrm{Vl}=$ valor de importância

\begin{tabular}{|c|c|c|c|c|c|c|c|c|c|c|c|c|c|c|}
\hline Nome Científico & Nome Vulgar & $\mathbf{N}$ & $\mathbf{U}$ & $A B$ & DA & DR & FA & FR & DoA & DoR & VC & VC (\%) & VI & VI (\%) \\
\hline Euterpe precatoria & Açaí & 82 & 3 & 1,31 & 136,7 & 13,8 & 100 & 8,3 & 2,19 & 39 & 52,8 & 26,4 & 61,1 & 20,4 \\
\hline Astrocaryum ulei & Murmuru & 133 & 3 & 1 & 221,7 & 22,3 & 100 & 8,3 & 1,67 & 29,7 & 52 & 26 & 60,4 & 20,1 \\
\hline Attalea phalerata & Uricuri & 84 & 3 & 1,04 & 140 & 14,1 & 100 & 8,3 & 1,73 & 30,8 & 44,9 & 22,4 & 53,2 & 17,7 \\
\hline Phytelephas macrocarpa & Jarina & 228 & 3 & 0 & 380 & 38,3 & 100 & 8,3 & 0 & 0 & 38,3 & 19,1 & 46,6 & 15,5 \\
\hline Bactris maraja & Maraje & 24 & 3 & 0 & 40 & 4,03 & 100 & 8,3 & 0 & 0 & 4,03 & 2,01 & 12,4 & 4,12 \\
\hline Oeno & Baca & 9 & 3 & 0,02 & 15 & 1,51 & 100 & 8,3 & 0,03 & 0,49 & 2 & 1 & 10,3 & 3,45 \\
\hline Desm & $\mathrm{Jac}$ & 11 & 3 & 0 & 8,33 & 1,85 & 100 & 8,3 & & 0 & 1,85 & 0,92 & 10,2 & 3,39 \\
\hline Socratea exorrhiza & Paxiubin & 6 & 2 & 0 & 10 & 1,01 & 66,7 & 5,6 & & 0 & 1,01 & 0,5 & 6,56 & 2,19 \\
\hline Bactris sp. & Marajá & 4 & 2 & 0 & 6,667 & 0,67 & 66,7 & 5,6 & & 0 & 0,67 & 0,34 & 6,23 & 2,08 \\
\hline Chelyocarpus chuco & Caranai & 4 & 2 & 0 & 6,667 & 0,67 & 66,7 & 5,6 & & 0 & 0,67 & 0,34 & 6,23 & 2,08 \\
\hline Attalea sp. & - & 2 & 2 & 0 & 3,333 & 0,34 & 66,7 & 5,6 & & 0 & 0,34 & 0,17 & 5,89 & 1,96 \\
\hline Bactris con & & 2 & 2 & 0 & 3,333 & 0,34 & 66,7 & 5,6 & & 0 & 0,34 & 0,17 & 5,89 & 1,96 \\
\hline Geon & & 2 & 1 & 0 & 3,333 & 0,34 & 33,3 & 2,8 & & 0 & 0,34 & 0,17 & 3,11 & 1,04 \\
\hline Iriarte & $\mathrm{Pa}$ & 2 & 1 & 0 & 333 & 0,34 & 33,3 & 2,8 & & & 0,34 & 0,17 & 3,11 & 1,04 \\
\hline Aipha & Pup & 1 & 1 & 0 & 1,667 & 0,17 & 33,3 & 2,8 & & 0 & 0,17 & 0,08 & 2,95 & 0,98 \\
\hline Bactr & $\mathrm{Ma}$ & 1 & 1 & 0 & 1,667 & 0,17 & 33,3 & 2,8 & ( & 0 & 0,17 & 0,08 & 2,95 & 0,98 \\
\hline Geonoma sp. & Ubim & 1 & 1 & 0 & 1,667 & 0,17 & 33,3 & 2,8 & v & 0 & 0,17 & 0,08 & 2,95 & 0,98 \\
\hline & ${ }^{\star \star *}$ Tot & 596 & 0 & .37 & 993.3 & 100 & 1200 & 100 & 5.61 & 100 & 200 & 100 & 300 & 100 \\
\hline
\end{tabular}




\section{Índice de similaridade de Jaccard (SJ)}

A matriz de similaridade de Jaccard relacionou as 17 espécies de palmeiras encontradas nas três parcelas distribuídas em suas respectivas parcelas. E possível observar na tabela 3 que as parcelas localizadas na área de platô e de encosta são as mais similares $(70 \%)$, ao passo que as áreas de baixio e encosta as menos similares (63,36\%). Estes resultados estão de acordo com GOULDING et al. (1988), que consideram as áreas de vertente (aqui denominada 'encosta') como um gradiente fisionomicamente mais semelhante ao platô.

HOPKINS (2005) reforça essa sugestão em estudo realizado na Reserva Ducke, em Manaus-AM, no qual ele observou que a composição da comunidade vegetal e a altura do dossel nas áreas de encosta são similares aos das áreas de platô. Este autor também considera que as florestas em áreas de vertentes se constituem em uma transição entre as áreas de platô e baixio. SILVA et al. (2014) verificaram maior similaridade entre parcelas instaladas em baixo e encosta na área da APA Igarapé São Francisco.

TABELA 3 - Matriz de similaridade de Jaccard entre as parcelas de palmeiras instaladas em áreas de baixio, encosta e platô na Reserva Florestal Humaitá, Porto Acre, Acre.

\begin{tabular}{lccc} 
& Baixio & Encosta & Platô \\
\hline Baixio & $*$ & $63,36 \%$ & $66,66 \%$ \\
Encosta & $*$ & $*$ & $70 \%$ \\
Platô & $*$ & $*$ & $*$ \\
\hline
\end{tabular}

\section{Índice de agregação das espécies}

A classificação dos indivíduos quanto à agregação mostra que 5 espécies $(29,41 \%)$ apresentaram tendência a agrupamento enquanto que 12 espécies $(70,59 \%)$ apresentaram agregação uniforme (Tabela 4). A espécie com maior índice geral de agregação (IGA) foi $S$. exorrhiza $(1,82)$, uma espécie comum em áreas inundáveis próximo de cursos de água (HENDERSON, 1995; SALM et al., 2015). A agregação de $S$. exorrhiza observada no presente estudo se explica pelo fato de a mesma ter sido encontrada apenas na área de baixio (4 indivíduos) e na área de encosta adjacente (2 indivíduos). A mesma explicação se aplica para as espécies $C$. chuco e $I$. deltoidea, frequentes em áreas inundáveis e encontradas, no presente estudo, apenas na área de baixio e de encosta.

TABELA 4 - Índice geral de agregação das espécies de palmeiras encontradas nas parcelas de baixio, encosta e platô na Reserva Florestal Humaitá, Porto Acre, Acre.

\begin{tabular}{|lcccc|}
\hline Nome Científico & Ui & Ut & IGA & Classif. IGA \\
\hline Socratea exorrhiza & 2 & 3 & 1,82 & Tend. Agrup. \\
\hline Chelyocarpus chuco & 2 & 3 & 1,21 & Tend. Agrup. \\
Bactris sp. & 2 & 3 & 1,21 & Tend. Agrup. \\
\hline Iriartea deltoidea & 1 & 3 & 1,64 & Tend. Agrup. \\
\hline Geonoma acaulis & 1 & 3 & 1,64 & Tend. Agrup. \\
\hline
\end{tabular}

ENCICLOPÉDIA BIOSFERA, Centro Científico Conhecer - Goiânia, v.11 n.22; p.286 2015 


\begin{tabular}{lllll} 
Bactris concinna & 2 & 3 & 0,61 & Uniforme \\
\hline Attalea sp. & 2 & 3 & 0,61 & Uniforme \\
\hline Aiphanes aculeata & 1 & 3 & 0,82 & Uniforme \\
\hline Geonoma sp. & 1 & 3 & 0,82 & Uniforme \\
\hline Bactris major & 1 & 3 & 0,82 & Uniforme \\
\hline Euterpe precatoria & 3 & 3 & ${ }^{*}$ & Uniforme \\
\hline Astrocaryum ulei & 3 & 3 & ${ }^{*}$ & Uniforme \\
\hline Attalea phalerata & 3 & 3 & ${ }^{*}$ & Uniforme \\
\hline Phytelephas macrocarpa & 3 & 3 & ${ }^{*}$ & Uniforme \\
\hline Bactris maraja & 3 & 3 & ${ }^{*}$ & Uniforme \\
\hline Oenocarpus mapora & 3 & 3 & ${ }^{*}$ & Uniforme \\
\hline Desmoncus mitis & 3 & 3 & ${ }^{*}$ & Uniforme \\
\hline
\end{tabular}

\section{Aspectos morfológicos das espécies}

A maioria dos indivíduos levantados em todas as áreas apresentou estipe com hábito solitário em razão das espécies mais abundantes, Phytelephas macrocarpa, Astrocaryum ulei, Attalea phalerata e Euterpe precatoria, que representaram $88,42 \%$ de todos os indivíduos encontrados, apresentarem esta característica (Figura 2A). Apesar disso, nove das 17 espécies encontradas apresentavam hábito cespitoso. Este tipo de hábito em palmeiras é considerado uma adaptação para suportar condições climáticas desfavoráveis, pois permite a reprodução assexuada das espécies, que, nos casos de estipe solitário, dependem exclusivamente da reprodução sexuada via sementes para sobreviver (BALSLEV et al. 2012).

Apenas uma espécie apresentou hábito escandente (Desmoncus mitis) e os indivíduos amostrados representaram apenas 1,84\% do total avaliado (Figura 2B). A presença de espinhos funciona como defesa das palmeiras contra herbívoros (BAKER \& COUVREUR, 2013). Os resultados do presente estudo demonstraram que espinhos estiveram presentes em sete das 17 espécies identificadas. Das espécies espinescentes, apenas duas eram de médio a grande porte $(A$. ulei e $A$. aculeata). As demais eram espécies de pequeno porte do subosque da floresta. De todos os indivíduos amostrados, $29,7 \%$ apresentaram espinhos, com destaque para os indivíduos do gênero Astrocaryum (Figura 2C). 

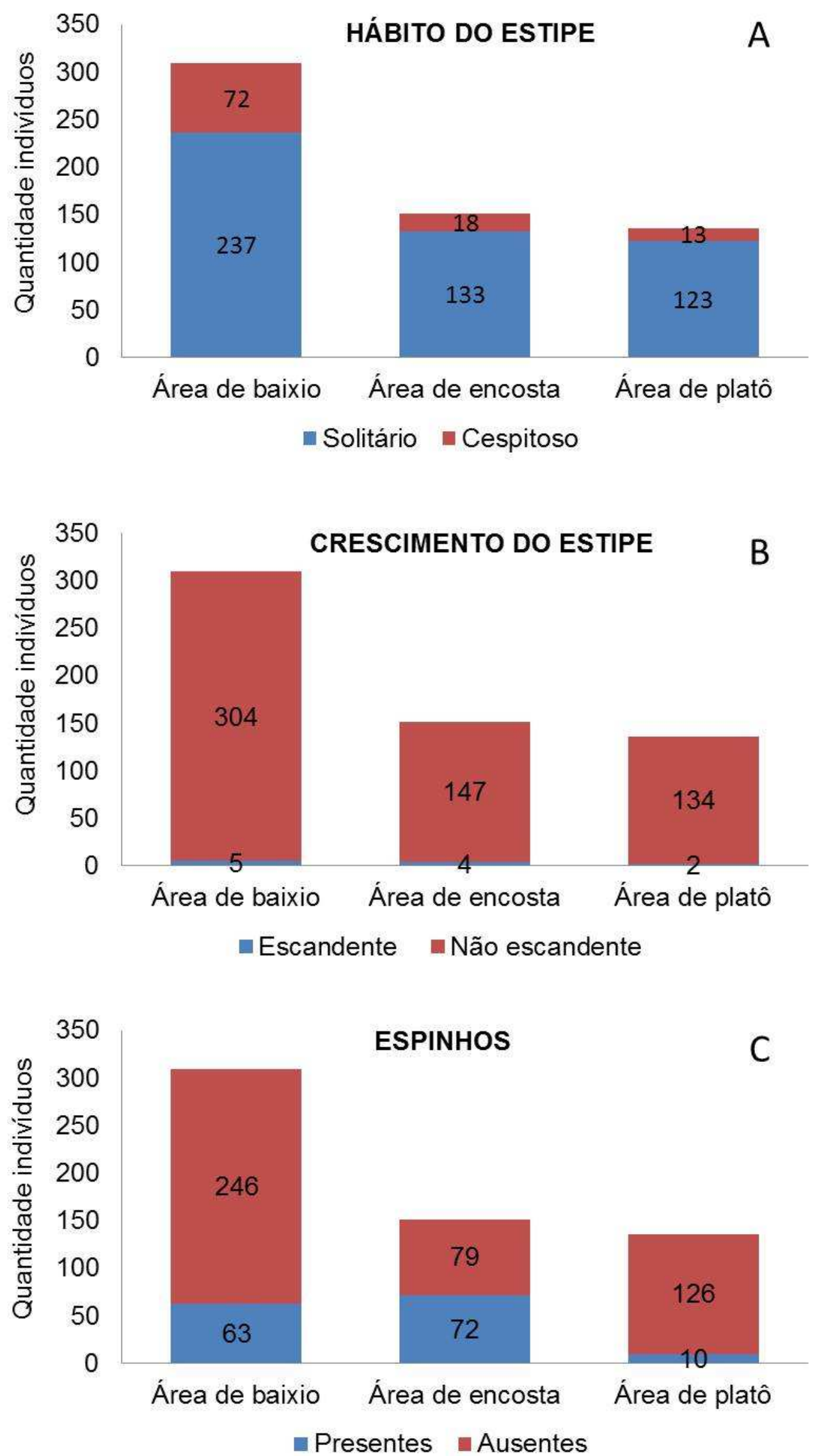

FIGURA 2 - Aspectos morfológicos das espécies de palmeiras avaliadas na Reserva Florestal Humaitá, Porto Acre, Acre: A - Hábito do estipe cespitoso ou solitário; B - Tipo de crescimento do estipe escandente ou não escandente; e $C$ - Presença ou ausência de espinhos. 


\section{CONCLUSÕES}

A maioria dos indivíduos de palmeiras foi encontrada na área de baixo que, apesar disso, revelou-se menos diversa que as demais áreas;

O gênero mais diverso foi Bactris e a espécie mais abundante Phytelephas macrocarpa. O fato de Euterpe precatoria ter apresentado maior VI reflete apenas a sua maior área basal, tendo em vista que foi apenas a quarta espécie mais abundante;

A maior similaridade florística entre as parcelas instaladas na área de platô e encosta reflete o fato da segunda ser considerada uma extensão florística e fisionômica da primeira;

A ampla distribuição da maioria das espécies resultou na predominância do padrão de agregação uniforme. A tendência ao agrupamento de algumas espécies, por outro lado, reflete a distribuição restrita das mesmas em uma ou no máximo duas áreas;

A presença de espinhos e estipes cespitosos da maioria das espécies de pequeno porte pode estar relacionada com 0 fato de as mesmas ocuparem 0 subosque da floresta, onde a competição por luz é mais intensa e o risco de predação por herbívoros mais elevado;

A estrutura populacional mostrou tendência ao ' $J$ ' invertido apenas na área de baixio, indicando um equilíbrio entre o recrutamento e a mortalidade de plantas nesta área.

\section{REFERÊNCIAS}

ACRE. Zoneamento Ecológico-Econômico do Acre. Recursos Naturais e Meio Ambiente, vol. 1. Rio Branco, Ministério do Meio Ambiente/Cooperação BrasilAlemanha/PPG-7. 2006.

BAKER, W. J.; COUVREUR, T. L. P. Global biogeography and diversification of palms sheds light on the evolution of tropical lineages. II. Diversification history and origin of regional assemblages. Journal of Biogeography, Maiden, USA, v.40, n.2, p.286-298, 2013.

BALSLEV, H.; EISERHARDT, W., KRISTIANSEN, T.; PEDERSEN, D. Palms and Palm Communities in the Upper Ucayali River Valley - a Little-Known Region in the Amazon Basin. Palms, Austin, USA, v. 54, n. 2, p. 57-72, 2010.

BALSLEV, H.; KAHN, F.; MILLAN, B.; SVENNING, J. C.; KRISTIANSEN, T.; BORCHSENIUS, F.; PEDERSEN, D.; EISERHARDT. Species diversity and growth forms in tropical American palm communities. Botanical Review, New York, v.77, n. 5, p. 381-425, 2011.

BALSLEV, H.; DURÁN, Z. P.; PEDERSEN, D.; EISERHARDT, W. L.; ASTURIZAGA, A. S.; ZAMBRANA, N. P. Subandean and adjacent lowland palm communities in Bolivia. Ecología en Bolivia, La Paz, v. 47, n.1, p.7-36, 2012.

BROWER, J. E.; ZARR, J. H. Field \& Laboratory Methods for General Ecology. lowa: Wm. C. Brown Company (2 nd ed.), 1984. 226p. 
CARVALHO, A. L.; FERREIRA, E. J. L.; LIMA, J. M. T. Comparações florísticas e estruturais entre comunidades de palmeiras em fragmentos de floresta primaria $e$ secundaria da APA Raimundo Irineu Serra, Rio Branco, Acre. Acta Amazonica, Manaus-AM, v.40, n. 4, p. 657-666, 2010.

CAMPOS, M. T.; EHRINGHAUS, C. Plant virtues are in the eyes of the beholders: a comparison of known palm uses among indigenous and folk communities of southwestern amazonia. Economic Botany, New York, v. 57, n. 3, p. 324-344, 2003.

DUARTE, A. F. Variabilidade e tendência das chuvas em Rio Branco, Acre, Brasil. Revista Brasileira de Meteorologia, São José dos Campos-SP, v. 20, p. 37-42, 2005.

EISERHARDT, W. L.; SVENNING, J. C.; KISSLING, W. D.; BALSLEV, H.; Geographical ecology of the palms (Arecaceae): determinants of diversity and distributions across spatial scales. Annals of Botany, Exeter, v.108, n. 8, p.13911416, 2011.

EMILIO, T. et al. Soil physical conditions limit palm and tree basal area in Amazonian forests. Plant Ecology \& Diversity, Edinburgh v. 7, n. 1-2, p. 215-229, 2014.

FERREIRA, E. J. L.. O bambu é um desafio para a conservação e o manejo de florestas no sudoeste da Amazônia. Ciência e Cultura, São Paulo, v. 66, n. 3, p.4651, 2014.

FERREIRA, E. J. L. Diversidade e importância econômica das palmeiras da Amazônia Brasileira. In: CONGRESSO NACIONAL DE BOTÂNICA, 56. 2005a. Curitiba, PR. Anais eletrônicos... Curitiba, PR. Sociedade de Botânica do Brasil, SBB. Disponível em: <http://www.botanica.org.br/trabalhoscientificos/56CNBot/56CNBot-2203.pdf >. Acesso em: 03 jun 2015.

FERREIRA, E. J. L. F. Manual das palmeiras do Acre, Brasil. 2005b. Disponível em: <http://www.nybg.org/bsci/acre/www1/manual_palmeiras.html>. Acesso em: 03 jun 2015.

GOULDING, M.; CARVALHO, M. L.; FERREIRA, E. G. Rio Negro, rich life in poor water. Haia: SPB Academic Publishing, 1988. 200pp.

HENDERSON, A. J. The palms of the Amazon. New York: Oxford University Press, 1995. 362p.

HOPKINS, M. J. G. Flora da Reserva Ducke, Amazonas, Brasil. Rodriguésia, Rio de Janeiro, v. 56, n. 86, p. 9-25, 2005.

LORENZI, H.; NOBLICK, L.; KAHN, F.; FERREIRA, E. J. L. Flora Brasileira: Arecaceae (Palmeiras). Nova Odessa: Plantarum, 2010. 384p. 
MAGURRAN, A. E. Ecological diversity and its measurement. New Jersey: Princeton University Press, 1988. 179p.

MARTINS, R. C. A. Família Arecaceae (Palmae) no Estado de Goiás: florística e etnobotânica. 2012. 292f. Tese (Doutorado em Botânica) - UNB, Brasília-DF, 2012. Disponível em:

<http://repositorio.unb.br/bitstream/10482/12165/1/2012_RenataCorreaMartins.pdf>. Acesso em: 30 set. 2015.

MESQUITA, M. L. R.; ANDRADE, L. A.; PEREIRA, W. E. Banco de sementes do solo em áreas de cultivo de subsistência na floresta ombrófila aberta com babaçu (Orbygnia phalerata Mart.) no Maranhão. Revista Árvore, Viçosa-MG, v. 38, n. 4, p.677-688, 2014.

MIRANDA, I. P. de A.; RABELO, A. Guia de identificação das palmeiras de Porto Trombetas, PA. Manaus: Editora da Universidade Federal do Amazonas, Instituto Nacional de Pesquisas da Amazônia, 2008. 365p.

MUELLER-DOMBOIS, D.; ELLENBERG, H. Aims and methods of vegetation ecology. New York: Willey \& Sons, 1974. 547p.

PETERS, C. M.; M. J. BALICK; F. KAHN; A. ANDERSON. Oligarchic forests of economic plants in Amazonia: Utilization and conservation of an important tropical forest resource. Conservation Biology, New York, USA, v.10, n.1, p.1-12, 1989.

PINHEIRO, C. U. B. Palmeiras do Maranhão: Onde canta o Sabiá. São Luís: Gráfica e Editora Aquarela, 2011. 232p.

RIBEIRO, J.E.L.S. et al. Flora da Reserva Ducke: Guia de identificação das plantas vasculares de uma floresta de terra-firme na Amazônia Central. Manaus: DFID-INPA, 1999. 816p.

ROCHA, A. E. S.; SILVA, M. F. F. Aspectos fitossociológicos, florísticos e etnobotânicos das palmeiras (Arecaceae) de floresta secundária no município de Bragança, PA, Brasil. Acta Botanica Brasilica, Belo Horizonte-MG, v.19, n. 3, p.657-667, 2005.

SALM, R.; PRATES, A.; SIMÕES, N. R.; FEDER, L. Palm community transitions along a topographic gradient from floodplain to terra firme in the eastern Amazon. Acta Amazonica, Manaus-AM, v. 45, n. 1, p.65-74, 2015.

SANTOS, E. A. et al.. Flora de palmeiras (Arecaceae) da área de influência da usina hidrelétrica de Jirau, Rondônia, brasil. IN: CONGRESO LATINOAMERICANO DE BOTÂNICA, 11; CONGRESSO NACIONAL DE BOTÂNICA, 65; ENCONTRO REGIONAL DE BOTÂNICOS MG, BA, ES, 34. 2014. Salvador, BA. Anais... Salvador, BA. Sociedade de Botânica do Brasil, SBB. CLBOT-CNABOT-ERBOT.

SILVA, G. M.; LIMA, A. F.; Mendonça, C. C.; SILVA, A. S.; Ferreira, E. J. L. Aspectos florísticos e fitossociológicos de comunidades de palmeiras (Arecaceae) em 
fragmentos florestais com e sem bambu (Guadua spp.) na APA Igarapé São Francisco, Acre. Enciclopédia Biosfera, Goiânia-GO, v.10, n.18, p.403-426, 2014.

SOUZA, A. L. Mata Nativa: sistema para a análise fitossociológica e elaboração de planos de manejo de florestas nativas, Viçosa: Consultoria de Desenvolvimento de Sistemas LTDA, 2006. 295p.

SOUZA, V. C.; LORENZI, H. Botânica sistemática: guia ilustrado para identificação das famílias de Angiospermas da flora brasileira baseado em APG II. 3 ed. Nova Odessa: Instituto Plantarum, 2012. 768 p.

TER STEEGE, H. et al. Hyperdominance in the Amazon tree flora. Science, v.342, p.325-334, 2013.

ZAMBRANA, N. Y. P.; BYG, A.; SVENNING, J. C.; MORAES, M.; GRANDEZ, C.; BALSLEV, $\mathrm{H}$. Diversity of palm uses in the western Amazon. Biodiversity and Conservation, Cham, Suiça, v. 16, n.10, p.2771-2787, 2007. 